\title{
Radiation-induced bowel injury: the impact of radiotherapy on survivorship after treatment for gynaecological cancers
}

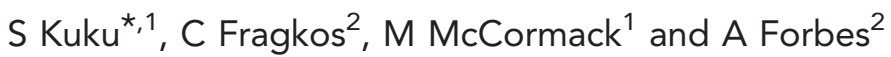 \\ ${ }^{1}$ Department of Oncology, University College London Hospitals, 250 Euston Road, London NW1 2PG, UK and ${ }^{2}$ Department of \\ Gastroenterology and Nutrition, University College London Hospitals, 250 Euston Road, London NW1 2PG, UK
}

Background: The number of women surviving cancer who live with symptoms of bowel toxicity affecting their quality of life continues to rise. In this retrospective study, we sought to describe and analyse the presenting clinical features in our cohort, and evaluate possible predictors of severity and chronicity in women with radiation-induced bowel injury after treatment for cervical and endometrial cancers.

Methods: Review of records of 541 women treated within the North London Gynaecological Cancer Network between 2003 and 2010 with radiotherapy with or without chemotherapy for cervical and endometrial cancer identified 152 women who reported significant new bowel symptoms after pelvic radiation.

Results: Factor analysis showed that the 14 most common and important presenting symptoms could be 'clustered' into 3 groups with predictive significance for chronicity and severity of disease. Median follow-up for all patients was 60 months. Univariate analysis showed increasing age, smoking, extended field radiation, cervical cancer treatment and the need for surgical intervention to be significant predictors for severity of ongoing disease at last follow-up. On multivariate analysis, only age, cancer type (cervix) and symptom combinations/'cluster' of (bloating, flatulence, urgency, rectal bleeding and per-rectal mucus) were found to be significant predictors of disease severity. Fifteen (19\%) women in the cervical cancer group had radiation-induced bowel injury requiring surgical intervention compared with five $(6.7 \%)$ in the endometrial cancer group.

Conclusion: Women with cervical cancer are younger and appear to suffer more severe symptoms of late bowel toxicity, whereas women treated for endometrial cancer suffer milder more chronic disease. The impact of radiation-induced bowel injury and the effect on cancer survivorship warrants further research into investigation of predictors of severe late toxicity. There is a need for prospective trials to aid early diagnosis, while identifying the underlying patho-physiological process of the bowel injury.

The significant increase in cancer survival rates over the last few decades have lead to more patients living with adverse effects of cancer treatments, which greatly affect their quality of life. Around one in ten of all women diagnosed with cancer in the UK will have a gynaecological cancer. Although cervical cancer is the nineteenth most common cancer in the UK accounting for about $2 \%$ of all new cancers in women, endometrial (uterine) cancer remains the most common gynaecological malignancy and fourth most common cancer in women (Cancer Research UK, Cancer statistics, 2010).
Pelvic radiation, as adjuvant treatment with or without chemotherapy in intermediate/high-risk endometrial cancer, and used concurrently with chemotherapy for locally advanced cervical cancer, remains a mainstay of multimodality treatment in gynaecological cancers. The number of long-term survivors has risen over the last few decades (Cancer Reseach UK, Cancer Satistics, 2010). Multimodality treatment has been shown in prospective randomised trials (Keys et al, 1999; Rose et al, 1999; Peters et al, 2000; Vale et al, 2010) to improve loco-regional disease 
control and overall survival, and with this we are seeing an increase in severe late effects in these women who live longer. The incidence of bowel symptoms that affect the quality of life in women after treatment remains under-reported (Andreyev, 2007a, b). This includes a change in bowel habits and symptoms but also lowgrade bowel and bladder symptoms that produce a cluster of 'small' problems; Klee et al (2000) reported a significantly higher incidence of low-grade diarrhoea in cervical cancer survivors 2 years after treatment compared with age-matched controls.

The true prevalence of late bowel toxicity remains unknown due to the paucity of prospective population studies in women who have received radiotherapy. Many patients are discharged from oncology centre follow-up after 2-5 years, and some are occasionally investigated and treated for bowel symptoms and complications in other units/hospitals. The reporting of late toxicity remains poor; many patients do not report mild chronic symptoms of loose stool and diarrhoea or those symptoms not considered to be related to treatment, or 'embarrassing' symptoms like flatulence and faecal incontinence/leaking. Although the majority of women who receive pelvic radiation will present with symptoms of acute radiation-induced bowel injury ('radiation enteritis/proctitis/proctopathy'), which may be present during and up to 3 months after treatment, some of these women report settlement of symptoms and a return to normal function (Andreyev, 2007a). It remains unclear why only a proportion of women (5-50\%, depending on study) (DeCosse et al, 1969; Galland and Spencer, 1985; Theis et al, 2010) never settle or indeed go on to represent months or years after pelvic radiation with symptoms of 'chronic radiation enteritis/proctitis/proctopathy'.

This radiation-induced injury to the small and/or large bowel is not simply dependent on radiation dose, fractions, amount of exposure and fields selected alone. There appears to be a complex interaction of patient, genetic and treatment factors that contribute to incidence, severity and chronicity of symptoms (Bentzen, 2006). The natural history of bowel injury had not been investigated well enough to offer evidence to explain why some women suffer from severe late effects and others do not, and indeed why some women appear to recover from symptoms (with or without intervention), whereas a significant proportion will carry on to have mild to moderate (sometimes tolerable) long-term symptoms. Late injury due to small vessel injury with endothelial damage, inflammation fibrosis, ischaemia and necrosis typically becomes symptomatic after a latent period between the end of acute effects and the development of late effects, but there may be a continuous progression from oedema, mucosal and submucosal inflammation and persistent ulceration to fibrosis (DeCosse et al, 1969). Clinically severe late radiation damage can present as strictures and stenoses with obstruction, fistulas and bowel perforation (Galland and Spencer, 1985).

The management of chronic radiation-induced bowel injury and presenting symptoms in patients remains challenging (Andreyev et al, 2011, 2012). The impact on womens' lives and cost to healthcare is evident. We aim to demonstrate our centre's experience and highlight the urgent need for prospective studies to better understand the patho-physiology of pelvic radiation disease and improve 'cancer survivorship'.

\section{METHODS}

Study cohort. The records of 541 patients diagnosed between February 2003 and June 2010 within the North London Cancer Gynaecological Cancer Network who required radiation as part of their treatment were reviewed; 219 patients with histologically confirmed cervical cancer - International Federation of Gynecology and Obstetrics (FIGO) stages IB2-IVA and 322 women with endometrial cancer FIGO-treated with radiotherapy with or without surgery and/or chemotherapy. Inclusion criteria included patients with both cancer types who had received a treatment dose of radiation according to standard protocols at our centre who then reported symptoms of bowel toxicity requiring investigation or referral to a gastroenterologist. Patients who presented with symptoms after 3 months from completion of radiotherapy were included in the study as well as those who reported 'chronic' symptoms which had not subsided or improved since the end of treatment. Exclusion criteria included patients who received a palliative dose (20-30 Gy) of radiation for recurrence or advanced disease and patients who reported mild symptoms after 3months post completion of radiotherapy, which had settled on the next subsequent follow-up.

Data collection. Our oncology and radiotherapy databases were searched to identify all patients who had received radiotherapy with curative intent. The gastroenterology database of patients referred from oncology as well as a database of the gastroenterologist with an interest in pelvic radiation disease were also searched to identify patients investigated for bowel symptoms after pelvic radiation. Clinical data on cancer demographics, treatment received, outcome and follow-up were obtained from hospital records. Original pathology reports were reviewed for histologic type, FIGO stage and tumour grade. We extracted data on the date of diagnosis, past medical and smoking history, date and type of primary surgery, stage of disease, type and dose of radiotherapy; date of completion of radiotherapy, chemotherapy received, disease recurrence or progression; date of presentation with bowel symptoms, nature of symptoms and degree to which symptoms affected quality of life (QoL), investigations and treatment of chronic radiation-related bowel symptoms, as well as status at last oncology and gastroenterology follow-up were all also obtained from individual patient records, clinic letters and review of imaging reports and other investigation results.

Patients were followed up in the multidisciplinary oncology clinics every 3 months for 2 years, then 6 monthly for 2 (early-stage endometrial) and 3 to 5 , and up to 10 years (locally/(advanced) stage cervical) depending on cancer type and stage. Clinical examination and routine screening bowel and bladder toxicity screen were undertaken and documented. Patients were referred to gastroenterologists if bowel symptoms were moderate and deemed to be affecting the patients' QoL or if they had symptoms that required investigations (for example, rectal bleeding). Computerised tomography or magnetic resonance imaging was undertaken if clinically indicated. Patients referred from neighbouring cancer units initially were followed up at the centre for 2 years and were then referred back to the units if there were found to be free of disease after 2 or more years.

\section{Treatment}

\section{Cervical cancer}

Chemotherapy: Majority of patients FIGO (IB-IVA) received concomitant chemo-radiation with weekly cisplatin $40 \mathrm{mg} \mathrm{m}^{-2}$.

Radiotherapy: Radiotherapy was given according to our defined cancer centre's protocol; concurrently with weekly cisplatin; almost all patients received primary radiotherapy stages (IB-IVA): external beam radiation to the pelvis $(50.4 \mathrm{~Gy}$ in 28 fractions over 5.5 weeks using $8-15 \mathrm{mV}$ photons) and intracavity brachytherapy using an intrauterine ovoid system (15 Gy in two fractions/HDR/ point A). Extended fields were used to treat PALN using an AP/PA field to a dose of $45 \mathrm{~Gy}$ in 25 fractions over 5 weeks. Where parametrial invasion or pelvic side-wall extension was evident, a further $5.04 \mathrm{~Gy}$ in three fractions was delivered to the pelvic side wall $(n=27,38 \%)$. 
Surgery: Patients were selected based on risk determined by clinical staging for laparoscopic para-aortic node dissection. Primary surgery was not indicated for locally advanced stage disease in the majority of patients.

\section{Endometrial cancer}

Surgery: Primary treatment for all patients involved a total hysterectomy with bilateral salpingo-oophorectomy with peritoneal washings.

Chemotherapy: When adjuvant chemotherapy was given, patients received six cycles of carboplatin AUC 5 and paclitaxel $175 \mathrm{mg} \mathrm{min}^{-2}$ every 3 weeks. This was usually within 4 weeks after the primary surgery.

Radiotherapy: Radiotherapy to the pelvis was delivered using external beam radiotherapy at a dose of $45 \mathrm{~Gy}$ in 25 fractions over 5 weeks with an additional $12 \mathrm{~Gy}$ in two fractions to the vaginal vault. Radiation treatment started immediately after recovery from surgery or chemotherapy if received, and was usually within 2-4 weeks of completion of chemotherapy.

Radiotherapy planning: Until 2006, radiotherapy was planned using orthogonal films and delivered using four orthogonal fields. From 2007, radiotherapy was delivered using three-dimensional conformal radiotherapy, planned using a dedicated computerised tomography planning scan and with IV contrast unless contraindicated. The planning target volume was defined as the clinical target volume (obtained from pretreatment magnetic resonance imaging and EUA) with a $10 \mathrm{~mm}$ expansion. The external beam radiation to the pelvis was delivered using a four-field (AP/PA and two lateral fields) arrangement. The conventional pelvic field extended from the top of L5 to the bottom of the obturator foramen or $2 \mathrm{~cm}$ below the lowest level of disease and laterally $1.5 \mathrm{~cm}$ beyond the bony pelvis. The lateral fields extended from the anterior border of the symphysis pubis to the $\mathrm{S} 2 / 3$ interspace posteriorly.

Data analysis. Comparison between patient and treatment groups was done using $\chi^{2}$-analysis for discrete variables. Student's $t$-test was used to compare means. Risk factors/predictors for severe late bowel injury were assessed using univariate and multivariate ordinal logistic regression. Survival data were analysed using Log-rank test and the Kaplan-Meier method was used to show time course and chronicity of late-bowel symptoms related to survival. Factor analysis was used to assess associations between the multiple presenting symptoms and correlate this with our dataset outcomes. Survival was defined from date of completion of cancer therapy to the date of last follow-up or death. Status of bowel injury symptoms/disease was defined as the interval from the first documented presentation with bowel symptoms to the date last seen by gastroenterologists or late toxicity status recorded by oncologists. A $P$-value $<0.05$ was considered significant. Statistical analyses were performed with SPSS version 19.0 (IBM, Armonk, NY, USA).

\section{RESULTS}

Patients. The clinico-pathologic variables and characteristics of patients are summarised in Table 1. Documented evidence was available for 152 women treated for gynaecological cancers $(n=77$; cervix, $n=75$; endometrial) at our oncology department who subsequently reported symptoms suggestive of radiation-induced bowel injury. Almost half of the patients with cervical cancer were treated for stage IIB disease (35 out of $77 ; 45.5 \%$ ) with moderately differentiated tumours; grade 2 (47 out of $77,62 \%$ ). Majority were
Table 1. Patient characteristics and cancer demographics; cervical and endometrial cancer

\begin{tabular}{|c|c|c|}
\hline Primary cancer & Cervical & Endometrial \\
\hline Total no. of patients reviewed & 219 & 322 \\
\hline $\begin{array}{l}\text { Patients presenting with significant symptoms of } \\
\text { chronic treatment-related bowel injury (\% of total) }\end{array}$ & 77 (35\%) & $75(23 \%)$ \\
\hline Median age (range) & $52(27-81)$ & $63(40-80)$ \\
\hline
\end{tabular}

\begin{tabular}{|l|c|c|}
\hline Stage & \multicolumn{3}{l|}{} \\
\hline$I^{\text {a }}$ & 11 & 32 \\
II & 51 & 23 \\
III & 10 & 18 \\
IV & 4 & 0 \\
Recurrence & 1 & 3 \\
\hline
\end{tabular}

\begin{tabular}{|l|c|c|}
\hline Grade & \multicolumn{2}{|l|}{} \\
\hline 1 & 4 & 3 \\
2 & 25 & 42 \\
3 & 10 & 25 \\
Unknown & 5 \\
\hline
\end{tabular}

\section{Histology}

Squamous cell

Adenocarcinoma

Adenosquamous

Small cell

Clear cell

Mixed $^{\mathbf{b}}$

Uterine serous papillary

MMMT

Smokers

\begin{tabular}{l|l}
57 & 0
\end{tabular}

0

61

0

0

1

6

3

Smokers

Past medical history

Bowel - IBS

Bowel - diverticular disease

Hypertension

Diabetes (type II)

Interval to presentation (months)

Abbreviations: IBS = irritable bowel syndrome; $\mathrm{MMMT}=$ malignant mixed mullerian tumour (carcinosarcoma).

${ }^{a}$ Stage I; IB1/2 (cervical), IB/C (endometrial)-FIGO 1988.

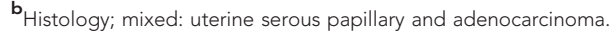

squamous cell carcinomas - 57 out of $77 ; 74 \%)$. Thirty-two (41.6\%) of the women with cervical cancer were smokers. Twelve (16\%) of the patients had a previous diagnosis of hypertension and two patients were type II diabetics, whereas four patients had been previously diagnosed with irritable bowel syndrome. Seventy-five patients treated for primary endometrial tumours were found to have reported new gastrointestinal symptoms thought to be radiation-induced. Majority of these women had stage IC (26 out of 75) and IIB (21 out of 75) endometrial cancers, grade 2 tumours (46 out of $75,61 \%$ ) and adenocarcinomas (61\%). Only five of the women reported as smokers. Four patients also had irritable bowel syndrome and five had a previous history of diverticular disease (none in the cervical cancer group). One of these women had had a previous bowel perforation related to diverticular disease (and one of only five women in the endometrial cancer group who needed surgery for resection of radiation-induced small bowel stricture). Median age was 52 years in the cervical cancer group and 63 years in the endometrial cancer group. 


\section{Treatment-cervical cancer}

Chemotherapy. Over $96 \%$ patients received five cycles of cisplatin or more. Sixteen (21\%) patients also received neo-adjuvant chemotherapy with 6 weeks of weekly carboplatin and paclitaxel within a clinical trial (McCormack et al, 2013). Two patients received carboplatin and etoposide for small-cell carcinomas. Two patients did not receive any chemotherapy due to elderly age and significant co-morbidities.

Radiotherapy. A total of 75 patients out of 77 received external beam radiotherapy $(50 \mathrm{~Gy})$ and intracavity bracytherapy as described in the methods. One patient, (stage IVA) underwent pelvic radiotherapy following hysterectomy: external beam radiation to the pelvis ( $45 \mathrm{~Gy}$ in 25 fractions over 5 weeks/10 MV photons) and vault brachytherapy ( $13 \mathrm{~Gy}$ in 2 fractions/HDR/ $0.5 \mathrm{~cm}$ from surface of applicator), whereas another (stage IVB) had consolidative pelvic radiotherapy $40.0 \mathrm{~Gy}$ in 5 fractions over 3 weeks after six cycles of cisplatin and topotecan for advanced disease.

Surgery. Fifteen women underwent laparoscopic para-aortic node dissection as part of staging. There was only one major perioperative complication; bowel injury due to a peri-operative complication with subsequent laparotomy with resection of perforated sigmoid and Hartmann's colostomy, this patient went on to suffer severe late toxicity requiring a right hemicolectomy. Only three women had surgery (total abdominal hysterectomy and bilateral salingoophorectomy) before (chemo)-radiation; two of these were incidental diagnoses on pathology.

\section{Treatment-endometrial cancer}

Chemotherapy. In all, 27 out of 75 (36\%) patients received 5 or 6 cycles of carboplatin and paclitaxel chemotherapy before radiation treatment.

Radiotherapy. All patients received external beam radiotherapy, as described in Methods.

Surgery. Forty-three patients underwent a total abdominal hysterectomy and bilateral salpingoophorectomy (TAH/BSO), whereas 32 patients had a laporoscopic procedure (TLH/BSO). One patient was an incidental pathological diagnosis on a vaginal hysterectomy specimen (done for prolapse). Surgical staging also involved pelvic and/or para-aortic lymphadenectomy in 14 patients. Five had an omentectomy as part of staging to exclude metastatic disease.

Presenting signs and symptoms of bowel injury after treatment. We identified 14 common 'new' bowel symptoms/signs reported by patients at follow-up and recorded; patients usually had multiple symptoms (Table 2). Patients who reported symptoms affecting QoL were referred to gastroenterologists; 90\% (69 out of 77) cervical cancer patients, and $83 \%$ (62 out of 75) of the endometrial cancer patients. Median time to presentation with bowel symptoms after completion of radiotherapy (or chemoradiation) was 8 months ( 1 month -9 years) in the cervical cancer group and 10 months ( 1 month -5 years) in women treated for endometrial cancer. Figure 1 shows the interval time to presentation (in oncology clinic) from completion of radiotherapy. There was no statistical difference in interval from completion of radiotherapy to presentation between cancer types.

Defecation urgency was the most common reported (and documented) symptom in 124 out of $152(81.6 \%)$ of women. Diarrhoea was reported in $54.6 \%$ (83 out of 152 ) of women; $30.3 \%$ of women reported increased frequency with bowels opening over four times a day (range 5-10). Twenty-eight women (18.4\% of the entire cohort) presented with or reported symptoms and signs of intermittent subacute bowel obstruction at oncology follow-up. Six

\begin{tabular}{|c|c|c|c|c|c|}
\hline & \multicolumn{4}{|c|}{ Patient group } & \multirow{3}{*}{$\begin{array}{c}\text { ALL } \\
(n=152) \\
n(\%)\end{array}$} \\
\hline & \multicolumn{2}{|c|}{$\begin{array}{l}\text { Cervical } \\
\text { cancer } \\
(n=77)\end{array}$} & \multicolumn{2}{|c|}{$\begin{array}{c}\text { Endometrial } \\
\text { cancer } \\
(n=73)\end{array}$} & \\
\hline & $n$ & $\begin{array}{l}\% \text { of } \\
\text { total }\end{array}$ & n & $\begin{array}{l}\% \text { of } \\
\text { total }\end{array}$ & \\
\hline Abdo pain & 38 & 49.4 & 34 & 45.3 & $72(47.4)$ \\
\hline Bloating & 22 & 28.6 & 23 & 30.7 & $45(29.6)$ \\
\hline Nausea & 12 & 15.6 & 7 & 9.3 & $19(12.5)$ \\
\hline Vomiting & 12 & 15.6 & 5 & 6.7 & $17(11.2)$ \\
\hline $\mathrm{SABO}^{\mathrm{a}}$ & 19 & 24.7 & 9 & 12.0 & $28(18.4)$ \\
\hline $\mathrm{ABO}^{\mathrm{b}}$ & 6 & 7.8 & 2 & 2.7 & $8(5.3)$ \\
\hline Diarrhoea & 47 & 61.0 & 36 & 48.0 & $83(54.6)$ \\
\hline $\mathrm{BO}<4$ per day & 51 & 66.2 & 49 & 65.3 & $100(65.8)$ \\
\hline $\mathrm{BO}>4$ per day & 26 & 33.8 & 20 & 26.7 & $46(30.3)$ \\
\hline Urgency & 62 & 80.5 & 62 & 82.7 & $124(81.6)$ \\
\hline Faecal incontinence/leaking & 26 & 33.8 & 16 & 21.3 & $42(27.6)$ \\
\hline Flatulence & 12 & 15.6 & 12 & 16.0 & $24(15.8)$ \\
\hline PR bleed & 28 & 26.4 & 28 & 37.3 & $56(36.8)$ \\
\hline PR mucus & 7 & 9.1 & 6 & 8.0 & $13(8.6)$ \\
\hline SAQOL & 32 & 41.6 & 28 & 37.3 & $60(39.5)$ \\
\hline
\end{tabular}

Abbreviations: $A L L=$ acute lymphoblastic leukemia; $B O=$ (frequency); number of times on average 'bowels opened'; $\mathrm{SAQOL}=$ patients reporting 'symptoms affecting their quality of life-'QoL'

${ }^{a} \mathrm{SABO}$-intermittent subacute bowel obstruction

${ }^{\mathbf{b}} \mathrm{ABO}$ - acute bowel obstruction presenting as emergency.

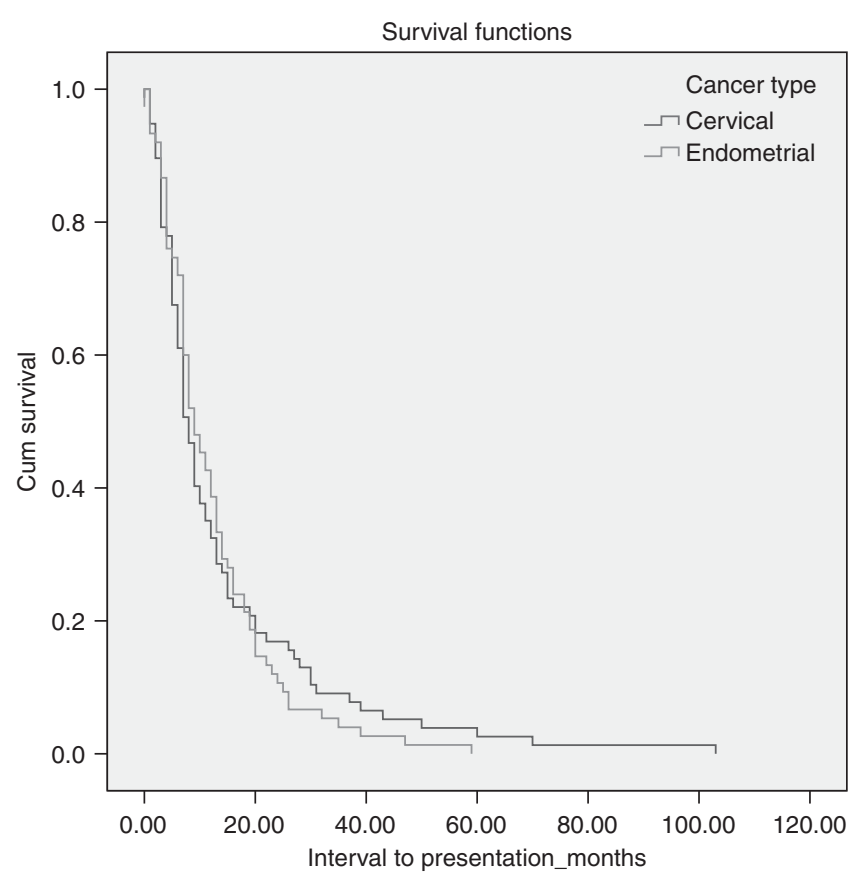

Figure 1. Interval to presentation with symptoms of radiation-induced bowel injury.

women in the cervical cancer group and two in the endometrial cancer group presented to emergency department with symptoms and signs of acute bowel obstruction. Forty-two (27.6\%) women reported faecal incontinence or admitted to leaking on questioning at gastroenterology assessment, whereas $36.8 \%(n=56)$ of women in the entire group reported rectal bleeding after radiotherapy. 
Table 3. Factor analysis; component loadings for presenting symptoms of bowel injury

\begin{tabular}{|c|c|c|c|c|c|c|c|}
\hline \multirow[b]{3}{*}{ Symptoms/signs } & \multicolumn{3}{|c|}{ Pattern matrix } & \multicolumn{3}{|c|}{ Structure matrix } & \multirow[b]{3}{*}{ Communalities } \\
\hline & \multicolumn{3}{|c|}{ Component } & \multicolumn{3}{|c|}{ Component } & \\
\hline & 1 & 2 & 3 & 1 & 2 & 3 & \\
\hline Nausea & 0.889 & & & 0.893 & & & 0.799 \\
\hline Vomitting & 0.869 & & & 0.874 & & & 0.767 \\
\hline SABO & 0.817 & & & 0.825 & & & 0.701 \\
\hline $\mathrm{ABO}$ & 0.547 & & & 0.548 & & & 0.352 \\
\hline $\mathrm{BO}>4 \mathrm{~d}$ & & 0.915 & & & 0.895 & & 0.794 \\
\hline $\mathrm{BO}<4 \mathrm{~d}$ & & -0.847 & 0.333 & & -0.808 & & 0.849 \\
\hline Diarrhoea & & 0.545 & & & 0.562 & & 0.34 \\
\hline $\mathrm{FI}$ & & 0.507 & & & 0.543 & 0.308 & 0.401 \\
\hline Bloating & 0.365 & & 0.695 & 0.354 & & 0.689 & 0.608 \\
\hline Flatulence & & & 0.589 & & & 0.591 & 0.373 \\
\hline Abdo pain & 0.517 & & 0.546 & 0.508 & & 0.537 & 0.555 \\
\hline Urgency & & 0.367 & 0.460 & -0.341 & 0.427 & 0.486 & 0.479 \\
\hline PRB & -0.303 & & -0.339 & & & -0.338 & 0.205 \\
\hline PR mucus & & & 0.339 & & & 0.336 & 0.124 \\
\hline \multicolumn{8}{|c|}{$\begin{array}{l}\text { Abbreviations: } \mathrm{ABO}=\text { acute bowel obstruction; } \mathrm{FI}=\text { faecal incontinence; } \mathrm{PRB}=\text { per-rectal bleeding; PR mucus = per-rectal mucus; } \mathrm{SABO}=\text { subacute bowel obstruction. Factor analysis tells us } \\
\text { what variables group or go together. Oblimin rotation generates both a pattern matrix and a structure matrix. The structure matrix is simply the factor loading matrix as in orthogonal rotation, } \\
\text { representing the variance in a measured variable explained by a factor on both a unique and common contributions basis. The pattern matrix, in contrast, contains coefficients which just } \\
\text { represent unique contributions (very similar to a correlation coefficient). For oblimin rotation, the researcher looks at both the structure and pattern coefficients when attributing a label to a } \\
\text { factor. By one rule of thumb, a factor loading level over } 0.3 \text { in absolute value is considered to indicate that this variable belongs to a factor; in any event, factor loadings must be interpreted in } \\
\text { the light of theory, not by arbitrary cutoff levels. In our case, we have highlighted with bold the loadings which indicated which variables where assigned to each component. Finally, the sum of } \\
\text { the squared factor loadings for all factors for a given variable (row) is the variance in that variable accounted for by all the factors, and this is called the communality. The communality measures } \\
\text { the percent of variance in a given variable explained by all the factors jointly and may be interpreted as the reliability of the indicator. }\end{array}$} \\
\hline
\end{tabular}

Factor analysis. As majority of patients presented with multiple symptoms, we sought to find correlation between symptoms in different subsets or 'clusters' of presenting symptoms. These items were subjected to principal components analysis to assess the suitability of data for factor analysis. Inspection of the correlation matrix revealed the presence of many coefficients 0.300 and above. The Kaiser-Meyer-Olkin value was 0.659 , exceeding the recommended value of 0.600 (Kaiser, 1974), and Bartlett's test of sphericity reached statistical significance (Bartlett, 1954), supporting the factorability of the correlation matrix. Principal components analysis (Armstrong and Soelberg, 1968) revealed the presence of three components, with eigen values exceeding one, explaining 24.1, 15.1 and $13.3 \%$. An inspection of the scree plot revealed a clear break after the third component. Using Cattell's (1966) scree test, we split the 'symptom clusters' into three components. This was further supported by the results of parallel analysis, which showed only three components, with eigen values exceeding the corresponding criterion values for a randomly generated data matrix of the same size (14 variables $\times 152$ respondents). The three-component solution explained a total of $52.5 \%$ of the variance. To aid in the interpretation of these, threecomponents oblimin rotation was performed. Factor loadings are shown in Table 3.

Component/Factor 1 can be labelled 'obstructive' symptoms, because it maintains high loading in symptoms suggestive of (radiotherapy-induced) bowel stricture/obstruction (nausea, abdominal pain, vomiting, signs and symptoms of intermittent subacute bowel obstruction and acute bowel obstruction). Component 2 - 'enteritis' symptoms (diarrhoea, loose stools and increased frequency, and faecal incontinence) suggests symptoms of small bowel dysfunction, whereas component 3 is more pathgnomonic, (though not definitive) of 'colitis-proctitis/proctopathy' symptoms (bloating, flatulence, abdominal pain, faecal urgency, per-rectal bleeding, and per-rectal mucus). Factor scores were computed and used in subsequent analyses. The purpose of this type of analysis and 'clustering' presenting symptoms was to
Table 4. Patient status at last follow-up

\begin{tabular}{|c|c|c|c|c|}
\hline & \multirow{2}{*}{\begin{tabular}{|c|} 
Cervical \\
n \\
\end{tabular}} & \multirow{2}{*}{\begin{tabular}{|c|} 
Endometrial \\
$n$
\end{tabular}} & \multicolumn{2}{|c|}{ Total } \\
\hline & & & $n$ & $\%$ \\
\hline $\begin{array}{l}\text { Alive and well, bowel symptoms } \\
\text { resolved } \\
\text { Alive with disease progression }\end{array}$ & $\begin{array}{l}21 \\
3\end{array}$ & 21 & $\begin{array}{r}42 \\
5\end{array}$ & $\begin{array}{r}27.6 \\
3.3\end{array}$ \\
\hline \multicolumn{5}{|c|}{ Alive, ongoing bowel symptoms } \\
\hline $\begin{array}{l}\text { Milda, managed with imodium/diet } \\
\text { Moderate }^{\mathbf{b}} \text {, awaiting further } \\
\text { investigations } \\
\text { Severe, symptoms affecting QoL }\end{array}$ & $\begin{array}{r}25 \\
4 \\
19\end{array}$ & $\begin{array}{r}38 \\
1 \\
2\end{array}$ & $\begin{array}{r}63 \\
5 \\
21\end{array}$ & $\begin{array}{r}41.4 \\
3.3 \\
13.8\end{array}$ \\
\hline \multicolumn{5}{|l|}{ Deceased } \\
\hline $\begin{array}{l}\text { Dead, recurrent disease } \\
\text { Dead, secondary to enteritis } \\
\text { complications } \\
\text { Dead, other causes }\end{array}$ & $\begin{array}{l}3 \\
1 \\
1 \\
5\end{array}$ & $\begin{array}{r}9 \\
0 \\
2 \\
11\end{array}$ & $\begin{array}{r}12 \\
1 \\
3 \\
16\end{array}$ & 10.5 \\
\hline $\begin{array}{l}\text { Abbreviation: } \mathrm{Q} \mathrm{OL}=\text { quality of life. } \\
\mathrm{a}_{\text {Mild; }} \text { urgency, frequency with loose sto } \\
\mathrm{b}_{\text {Moderate; patients requiring regul }} \\
\text { gastroenterologists +/colorectal surgeor }\end{array}$ & $\begin{array}{l}\text { Is }<4 \text { per day } \\
\text { follow-up }\end{array}$ & nd further invest & & with \\
\hline
\end{tabular}

try to identify, within our cohort of women, associations and possible predictors of disease course and severity, site of bowel injury and underlying patho-physiological processes.

Predictors of severity and chronicity of bowel problems. Patients were followed up until symptoms resolved or until their last oncology/gastroenterology follow-up before the end of data collection in December 2012. Information on status of bowel 


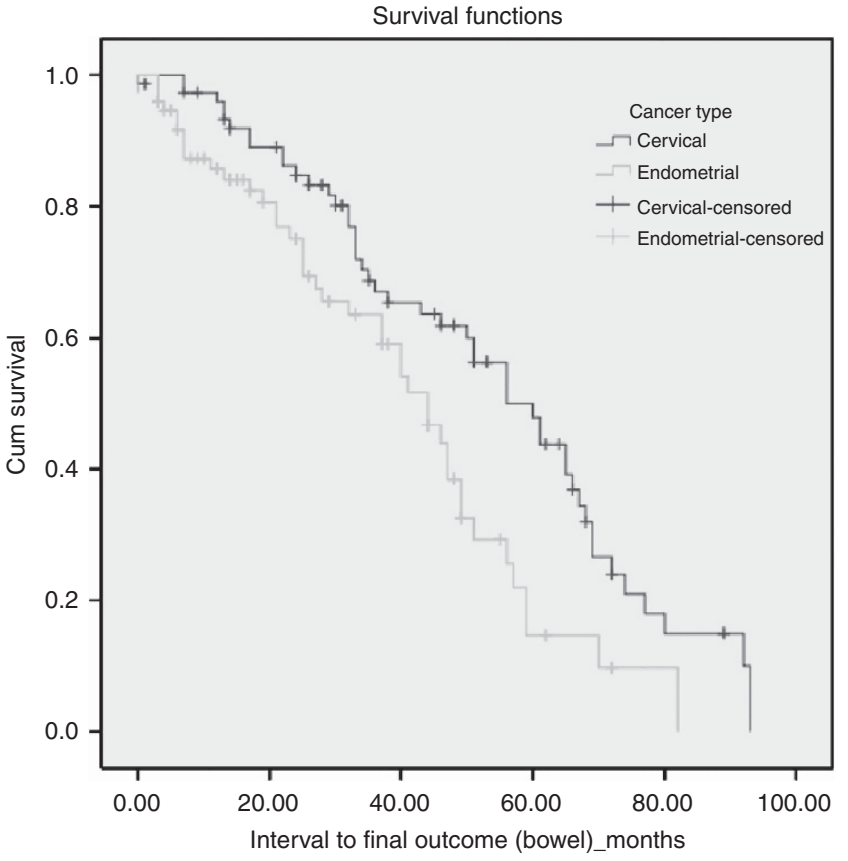

Figure 2. Follow-up of patients with bowel symptoms.

symptoms, (and disease/general health) was obtained from documentation at these consultations. Table 4 shows a summary of documented status at last follow-up. At last follow-up, 42 out of $152(27.6 \%)$ of women were alive, disease-free with resolution of bowel symptoms. Five patients $(3.3 \%)$ were alive with disease progression, without clear documentation of follow-up of late bowel toxicity symptoms. Sixty-three out of $152(41.1 \%)$ of all patients were disease-free with ongoing mild symptoms managed by oncologists and/or discharged from gastroenterology follow-up. Majority of these patients had mild urgency and frequency with loose stools, with bowel motions on an average twice a day. Most patients reported stable symptoms not affecting QoL and some required antimotility drugs (imodium) intermittently with dietary manipulation to control symptoms. Five women (3.3\%) had ongoing moderate symptoms, with significant diarrhoea, frequency and urgency and were undergoing further investigations. Twenty-one patients $-13.8 \%$ of patients still had ongoing severe symptoms affecting their QoL, some even after surgical intervention. Only two of these patients had been treated for endometrial cancer, whereas 19 had received concurrent chemo-radiation for a cervical primary cancer. Median follow-up time (from presentation with symptoms of radiation-induced bowel injury) was 89 months for the patients who had resolution of their symptoms at last followup; 37.75 months for patients with mild manageable symptoms; 50.5 months for the five patients with ongoing moderate symptoms (mostly urgency and diarrhoea); and 34.5 months for patients with ongoing severe symptoms of radiation-induced bowel injury at last follow-up. There was no statistical significant difference when comparing follow-up time and status (resolution or degree of severity) at last follow-up. Figure 2 shows the follow-up time from presentation with bowel symptoms. We found a significant difference between cancer types (Log-rank $\chi^{2}=8.065, P=0.005$ ) suggesting women with endometrial cancer were more likely to have less severe, but more chronic symptoms.

In univariable ordinal logistic regression analysis (Table 5), significant predictors of increasing severity of symptoms (from presentation to bowel status at last follow-up) were age, cancer type (cervical cancer; concurrent chemo-radiation), bowel injury requiring surgical intervention and symptom cluster/factor 3 that is, patients presenting with predominantly bloating, flatulence,
Table 5. Univariate (unadjusted) ordinal regression analysis showing predictors of increasing chronic radiation enteritis/proctitis severity in the whole cohort

\begin{tabular}{|l|c|c|}
\hline Variable & OR (95\% Cl) & $P$-value \\
\hline Age & $0.96(0.94-0.98)$ & 0.001 \\
Cancer type & $0.49(0.26-0.93)$ & 0.028 \\
(endometrial vs cervical) & $1.57(0.64-3.96)$ & 0.312 \\
History of diabetes/hypertension & $1.52(0.53-4.31)$ & 0.436 \\
History of previous IBS/diverticular disease & $0.32(0.17-0.77)$ & 0.008 \\
Smoking history & $0.76(0.42-1.36)$ & 0.356 \\
Stage of disease (cervix) & $0.78(0.45-1.36)$ & 0.388 \\
Stage of disease (endo) & $1.58(0.41-2.50)$ & 0.976 \\
NACT pre-chemoradiation (cervix) & $0.37(0.14-0.96)$ & 0.041 \\
Extended field RT (pelvic side wall boost/pa & & \\
strip) (cervix) & $0.59(0.21-1.65)$ & 0.311 \\
Laparoscopic vs open hysterectomy (endo) & $0.95(0.34-2.63)$ & 0.921 \\
Chemo vs no chemo (endo) & $2.13(0.58-7.75)$ & 0.255 \\
P/PA node dissection (endo) & $0.67(0.24-1.85)$ & 0.442 \\
P/PA node dissection (cervix) & $0.22(0.08-0.55)$ & 0.001 \\
Surgical treatment (no vs yes) & \multicolumn{2}{|l|}{} \\
\hline 'Symptom cluster' at presentation & $1.18(0.86-1.63)$ & 0.302 \\
& $1.10(0.80-1.51)$ & 0.548 \\
\hline Factor 1 & $1.36(1.00-1.86)$ & 0.053 \\
\hline Factor 2 & \multicolumn{2}{|l}{} \\
Factor 3 &
\end{tabular}

Abbreviations: $\mathrm{Cl}=$ confidence interval; $\mathrm{NACT}=$ neo-adjuvant chemotherapy; $\mathrm{OR}=$ odds ratio; $\mathrm{P} / \mathrm{PA}=$ pelvic/para-aortic node dissection; $\mathrm{RT}=$ Radiotherapy

Table 6. Multivariate (adjusted) ordinal regression analysis showing predictors of increasing chronic radiation enteritis/proctitis severity in the whole cohort

\begin{tabular}{|l|c|c|c|c|}
\hline \multicolumn{2}{|c|}{} & \multicolumn{3}{c|}{$\begin{array}{c}95 \% \text { Confidence } \\
\text { interval }\end{array}$} \\
\hline & OR & Lower & Upper & P-value \\
\hline Age & 0.97 & 0.94 & 1.00 & 0.050 \\
\hline Smoking & 0.42 & 0.18 & 0.98 & 0.044 \\
\hline 'Symptom cluster'/factor 3 & 1.51 & 1.08 & 2.11 & 0.017 \\
\hline $\begin{array}{l}\text { Cancer type } \\
\text { (cervical vs endometrial) }\end{array}$ & 1.21 & 0.55 & 2.63 & 0.637 \\
\hline Surgery (no vs yes) & 0.23 & 0.09 & 0.65 & 0.005 \\
\hline Abbreviation: OR, odds ratio. & & & & \\
\hline
\end{tabular}

abdominal pain, faecal urgency, per-rectal bleeding and per-rectal mucus. In multivariate ordinal regression analysis (Table 6), younger age (odds ratio: $0.97,95 \%$ confidence interval: $0.94-1.0$, $P=0.05$ ), smoking history (odds ratio: 0.42 , $95 \%$ confidence interval: $0.18-0.98, P=0.044$ ), surgical intervention (odds ratio: $0.23,95 \%$ confidence interval: $0.08-0.62, P=0.005)$ and initial presentation with 'symptom cluster'/factor 3 (odds ratio: 1.51, 95\% confidence interval: $1.08-2.11, P=0.017$ ) were independent predictors of severity of bowel symptoms, after correcting for cancer type.

Investigations and non-surgical therapeutic interventions for chronic radiation enteritis/proctitis in our cohort. Initial assessment of patients presenting with bowel symptoms after pelvic radiation was by the oncologists. Patients who typically had mild stable symptoms of radiation enteritis (diarrhoea alternating 
with loose stool, faecal urgency and increased number of motions per day $(\mathrm{BO}<4)$ were usually monitored with dietary manipulation advice and antidiarrhoeal agents imodium and/or codeine phosphate prescribed. Patients were offered referral to gastroenterologists if they had symptoms that were affecting their quality of life or if symptoms worsened. Patients who presented with rectal bleeding, urgency with faecal incontinence or any combination of symptoms which the patient deemed 'affecting quality of life' were immediately referred to a gastroenterologist.

Routine bloods tests to exclude anaemia and vitamin B12 deficiency were done for all patients presenting to gastroenterology. The most common investigation was a colonoscopy (and biopsy of any abnormal areas); 73 out of 152 (48\%) women underwent a colonoscopy, whereas 22 women (14.5\%) had a flexible sigmoidoscopy. Forty (40 out of 73) of these patients had clear endoscopic evidence of distal colitis/proctosigmoiditis and/or telangiectasia/angioma attributed to radiation changes to bowel mucosa and documented on endoscopy findings.

Women had imaging; either an magnetic resonance imaging to exclude disease recurrence as a cause for symptoms or a computerised tomography scan, usually if bowel obstruction was suspected. Radiological imaging was useful in supporting diagnoses by demonstrating dilated and/or thickened small or large bowel loops and radiation-related strictures, as well as bladder wall and rectal thickening and oedema. Endo-anal ultrasound scans and ano-rectal physiology testing were performed to exclude sphincter defects in women presenting with incontinence/leaking. From 2010, a small proportion of patients had a SeCHAT scan to exclude bile acid malabsorption as a potential cause for ongoing diarrhoea, whereas 14 women required a hydrogen breath test to exclude small bowel bacterial overgrowth.

We analysed investigations relating to the three presenting symptom clusters/factors derived from factor analysis. There was a significant difference $P<0.05$ in the proportion of patients presenting with symptom cluster/factor 1 (obstructive) who went on to have an magnetic resonance imaging/computerised tomography compared with those who scored higher for factors 2 and 3. Patients who had an endo-anal ultrasound and ano-rectal physiology as part of work-up for presenting symptoms of bowel toxicity were more likely to have scored for factor 2 compared with factor $1 / 3(P<0.001)$. There was no significant difference between the three symptom clusters/factors for patients who underwent flexible sigmoidoscopy or colonoscopy.

The use of antimotility/antidiarrhoeal drugs was the most common initial intervention in most patients - $62.5 \%$ of all patients needed imodium either regularly or as required for a period. Only 10 patients had documented evidence of requiring codeine phosphate as well as imodium to control diarrhoea/ frequency of motions. The trial of interventions varied over time and clinician, and was individualised based on symptoms and patients' investigation results. Before 2005, some patients received trials of steroid treatments; predsol enema, predfoam, hydrocortisone suppositories. Other treatments tried included: mebeverine $(n=3)$, activated charcoal $(n=2)$, asacol suppositories $(n=1)$, colesevalam and cholestyramine when bile acid malabsorption was suspected $(n=6)$, trial of antibiotics when small bowel bacterial overgrowth was diagnosed $(n=5)$, biofeedback to manage faecal incontinence $(n=5)$, and hyperbaric oxygen therapy under a clinical trial (HOT II; Institute of Cancer Research and Royal Marsden ISRCTN 86894066) $(n=3)$. Thirty-two patients in total (16 each in both cancer groups) required argon beam coagulation therapy and colonoscopy to manage rectal bleeding; eight of these patients required multiple (2 out of 3 ) procedures to control bleeding. Four women were treated for transfusion-dependent rectal bleeding, with repeat blood transfusions and argon beam coagulation.

\begin{tabular}{|l|c|c|}
\hline \multicolumn{3}{|c|}{ Table 7. Surgical management of radiation-induced bowel injury } \\
\hline & $\begin{array}{c}\text { Cervical } \\
\text { cancer }\end{array}$ & $\begin{array}{c}\text { Endometrial } \\
\text { cancer }\end{array}$ \\
\hline Surgical procedure & $\mathbf{n}$ & $\mathbf{n}$ \\
\hline Small bowel resection + adhesiolysis & $\mathbf{7}$ & $\mathbf{2}$ \\
\hline Primary anastamosis & 2 & 2 \\
Ileostomy & 1 & 0 \\
Reversal of ileostomy & 4 & 0 \\
\hline lleocaecal resection & 2 & 1 \\
\hline Right hemicolectomy & $\mathbf{4}$ & $\mathbf{2}$ \\
\hline Primary anastamosis & 2 & 1 \\
\hline Colostomy formation & 2 & 1 \\
\hline End colostomy formation (bypass) & 3 & 0 \\
\hline Adhesiolysis only & $\mathbf{1}$ & 0 \\
\hline Sigmoid stricture dilatation & $\mathbf{1}$ & 0 \\
\hline a Previous small bowel resection and re-obstruction. & \\
\hline
\end{tabular}

Surgical management of radiation-induced bowel injury. Table 7 shows the type of surgical treatment received by the women in our cohort who suffered severe late toxicity. Twenty (20 out of 152; $13.2 \%$ ) required surgical intervention to manage severe late radiation-induced toxicity. Four women required (ongoing) home parenteral nutrition after surgery to maintain nutrition, whereas seven women still had ongoing enteritis/colitis/obstructive symptoms affecting QoL, even after surgical resection/bypass. One patient was treated with repeated sigmoid dilatation (2006) for symptoms of intermittent subacute bowel obstruction with subsequent resolution of symptoms.

On univariate analysis, only cancer type (cervix) was associated with an increased risk of requiring surgery. There was no significant association found with tumour stage, smoking, past history of irritable bowel syndrome, divertucular disease, diabetes or hypertension, the use neo-adjuvant chemotherapy, extended field radiation-pelvic side wall $+/-$ a para-aortic boost. In endometrial cancer patients, type of primary surgery received, open $v s$ laparoscopic or whether patients received adjuvant chemotherapy before radiation or not were also not found to be significant predictors of severe late toxicity in our cohort. Although smoking was not found to be a statistically significant predictor of severe late toxicity requiring surgery, of six women reported heavy smoking on diagnosis $>20$ per day; 5 out of 6 of these women required surgical resection of radiation-damaged bowel whereas the one remaining patient had ongoing severe symptoms of enteritis and required surgery to repair a radiation-induced femoral occlusion.

\section{DISCUSSION}

The aim of this study was to describe and analyse the nature of presenting symptoms of bowel injury thought to be directly related to radiation treatment in our cohort of women and to determine associations with severity and chronicity of symptoms. Data was clinician-reported and collected retrospectively from patient notes. It is important to acknowledge not only that the reported number of cases and the proportion of women who seek help are only a fraction of true prevalence (Gami et al, 2003; Andreyev et al, 2005), but also that this still underestimates the actual frequency of pathologic changes and 'bowel injury', given some women may be 
asymptomatic and symptoms do not always correlate with disease activity directly related to radiation changes. Studies (Olopade et al, 2005; Khalid et al, 2006; Wedlake et al, 2010) using patientreported symptom tools, validated/modified questionnaires have shown that this method of collecting toxicity data to be more sensitive in characterising symptoms than clinician-based reporting without toxicity scores/questionnaires. Yet others (West and Davidson, 2009) acknowledge, that outside of the clinical trial setting these questionnaires/scoring tools (especially the CTCAEv3), are impractical in the clinical setting. Barraclough et al (2012), in a prospective analysis of patient-reported late toxicity in gynaecological cancers ( $73 \%$ cervix), had 126 out of 226 (56\%) patients withdraw from their study, with 60 patients discontinuing completion of questionnaires at various points throughout the study.

We appreciate that it is always difficult to make concrete conclusions from retrospectively collected data, yet this summary of our centre's experience gives some insight into the current practice and highlights the significant proportion of women who suffer symptoms affecting their quality of life persisting with varying severity and chronicity. What is clear is that patients present with 'clusters' of symptoms and this data, if collected prospectively and systematically at oncology follow-up, over time points, may well lead to a better understanding of the clinical course and predictors of severity of bowel symptoms.

We demonstrated the nature of symptoms reported and recorded in patient records and found fecal urgency to be the most commonly reported (and documented) symptom, in keeping with the literature (Denham et al, 1999; Gami et al, 2003; Putta and Andreyev, 2005; Andreyev et al, 2010). Multivariate analysis in our study showed that younger age, treatment for cervical cancer and presenting with the multiple symptom 'cluster' that included bloating, flatulence, abdominal pain, fecal urgency, rectal bleeding and rectal mucus were associated with more severe ongoing symptoms at follow-up (median 34.5 months). Capp et al (2009) have best defined symptom clusters for rectal toxicity in men treated for prostate cancer with data obtained through modified self-assessed questionnaires and follow-up at 1,2 and 3 years after radiotherapy. This study highlights the flaws within the CTC (common toxicity criteria) grading scale, especially in identifying rectal injury. The clusters identified (made up of only eight individual components/symptoms - compared with our 14) at different time points, also show faecal urgency to be at the 'core' of all symptom clusters. What remains unclear is how the various symptom clusters identified relate to underlying radiation-induced patho-physiological processes.

Barraclough et al (2012) in their study, use factor analysis to identify the most important questions likely to account for interpatient variability in subjective toxicity using a disease site-specific questionnaire developed from the LENT SOMA scales to score patient data. Both studies highlight the need for a better guide for clinicians in assessing patients at initial presentation (and before radiotherapy). It is our belief that more data available to test the reproducibility of the symptom cluster approach and the degree of severity of each symptom may well help to better identify the type and site of the underlying radiation-induced bowel injury pathological processes.

We demonstrated a prevalence of $\sim 13 \%$ of women in our cohort with severe late bowel injury requiring surgical intervention, highlighting the associated morbidity and the need for further research to try to identify these patients at risk and offer early interventions. We recognise a number of limitations in our study. Data was collected by one single researcher to ensure consistency in extracting data and symptoms as recorded in records. All relevant correspondence, letters, investigations and results related to each patient were reviewed. Patients with incomplete or no follow-up documentation were excluded $(n=22)$. Collecting clinician-reported data retrospectively relies on documentation of different clinicians with varying views of symptoms and experience dealing with patients' bowel toxicity and assessment, which all may be influenced by the patients' clinical situation. It is impossible to correct for under-reporting and lack of documentation especially for those symptoms that may have been deemed 'less serious' by clinicians. There is also the question of whether bowel toxicity is more likely to be reported and recorded in 'healthier' survivors. Patients with disease recurrence and progression are less likely to report symptoms that may be related to radiation-induced bowel injury as they are no longer focused on symptoms of toxicity but rather worrying about symptoms related to their cancer progression.

As this was a retrospective study, there was no data identifying women's bowel function before radiotherapy, however, a thorough past medical history, including any reported abnormal bowel symptoms was routinely obtained from each patient at our centre before treatment and recorded on a 'front sheet'. All symptoms reported at initial presentation, time of referral to gastroenterology and on first consultation with gastroenterology were recorded. Analysis of clinical features did not take into account the grading/ severity of each symptom - for example the degree of urgency in combination with other symptoms in a 'cluster' might indeed represent different underlying patho-physiological processes for two different patients.

Prospective studies are urgently needed to better understand the natural history of radiation-induced bowel injury to guide the development of objective biomarkers of toxicity, a standard in assessing degree of toxicity to aid diagnostic pathways, and identification of the underlying patho-physiological processes leading to specific bowel injury. Our study highlights the important role of all clinicians, oncologists, gynaecologists and gastroenterologists in follow-up - survival and survivorship. It is important as clinicians that we find a structured, practical and universal approach in screening for and assessing symptoms of radiationinduced bowel injury.

\section{REFERENCES}

Andreyev HJN, Vlavianos P, Blake P, Dearnaley D, Norman AR, Tait D (2005) Gastrointestinal symptoms after pelvic radiotherapy: role for the gastroenterologist? Int J Radiat Oncol Biol Phys 62: 1464-1471.

Andreyev HJ (2007a) Gastrointestinal symptoms following therapeutic pelvic radiotherapy: a new understanding to improve the management of symptomatic patients. Lancet Oncol 8(11): 1007-1017.

Andreyev HJ (2007b) Gastrointestinal problems after pelvic radiotherapy; the past, the present and the future. Clin Oncol ( $R$ Coll Radiol) 19: 790-799.

Andreyev HJ, Wotherspoon A, Denham JW, Hauer-Jensen M (2010) Defining pelvic-radiation disease for the survivorship era. Lancet Oncol 11: $310-312$.

Andreyev HJN, Wotherspoon A, Denham JW, Hauer-Jensen M (2011) "Pelvic radiation disease": New understanding and new solutions for a new disease in the era of cancer survivorship. Scand J Gastroenterol 46: 386-397.

Andreyev HJ, Davidson SE, Gillespie C, Allum WH, Swarbrick E (2012) Practice guidance on the management of acute and chronic gastrointestinal problems arising as a result of treatment for cancer. Gut 61: 179-192.

Armstrong JS, Soelberg P (1968) On the interpretation of factor analysis. Psychol Bull 70: 361-364.

Barraclough LH, Routledge JA, Farnell D, Burns M, Livsey JE, Davidson SE (2012) Prospective analysis of patient-reported late toxicity following pelvic radiotherapy for gynaecological cancer. Radiother Oncol 103: 327-332.

Bartlett MS (1954) A note on the multiplying factors for various X2 Approximations. J R Stat Soc Series B Stat Methodol 6: 296-298. 
Bentzen SM (2006) Preventing or reducing late side effects of radiation therapy: radiobiology meets molecular pathology. Nat Rev Cancer 6: 702-713.

Cancer Research UK, Cancer statistics (2010) www.cancerresearchuk.org/ cancer-info/cancerstats.

Capp A, Inostroza-Ponta M, Bill D, Moscato P, Christie D, Lamb D, Turner S, Joseph D, Matthews J, Atkinson C, North J, Poulsen M, Spry NA, Tai K, Wynne C, Duchesne G, Steigler A, Denham JW (2009) Is there more than one proctitis syndrome? A revisitation using data from the TROG 96.01 trial. Radiother Oncol 90: 400-407.

Cattell RB (1966) The score test for the number of factors. Multivariate Behav Res 1: 245-276.

DeCosse JJ, Rhodes RS, Wentz WB, Reagen HJ, Dworken HJ, Holden WD (1969) The natural history and management of radiation induced injury of the gastrointestinal tract. Ann Surg 170: 369-384.

Denham JW, O'Brien PC, Dunstan RH, Johansen J, See A, Hamilton CS, Bydder S, Wright S (1999) Is there more than one late proctitis syndrome? Radiother Oncol 51: 43-53.

Galland RB, Spencer J (1985) The natural history of clinically established radiation enteritis. Lancet 1: 1257-1258.

Gami B, Harrington K, Blake P, Dearnaley D, Tait D, Davies J, Norman AR, Andreyev HJ (2003) How patients manage gastrointestinal symptoms after pelvic radiotherapy. Aliment Pharmacol Ther 18: 987-994.

Kaiser H (1974) An index of factoral simplicity. Psychometrika 39: 31-36.

Keys HM, Bundy HN, Stehman FB, Muderspach LI, Chafe WE, Suggs 3rd CL, Walker JL, Gersell D (1999) Cisplatin, radiation, and adjuvant hysterectomy compared with radiation and adjuvant hysterectomy for bulky stage IB cervical carcinoma. $N$ Engl J Med 340: 1154-1161.

Khalid U, McGough C, Hackett C, Blake P, Harrington KJ, Khoo VS, Tait D, Norman AR, Andreyev HJ (2006) A modified inflammatory bowel disease questionnaire and the vaizey incontinence questionnaire are more sensitive measures of acute gastrointestinal toxicity during pelvic radiotherapy than RTOG grading. Int J Radiat Oncol Biol Phys 64: 1432-1441.

Klee M, Thranov I, Machin D (2000) The patients' perspective on physical symptoms after radiotherapy for cervical cancer. Gynecol Oncol 76: 14-23.
McCormack M, Kadalayil L, Hackshaw A, Hall-Craggs MA, Symonds RP, Warwick V, Simonds H, Fernando I, Hammond M, James L, Feeney A, Ledermann JA (2013) A phase II study of weekly neoadjuvant chemotherapy followed by radical chemoradiation for locally advanced cervical cancer. Br J Cancer 108: 2464-2469.

Olopade FA, Norman AR, Blake P, Dearnaley DP, Harrington KJ, Khoo V, tait D, Hackett C, Andreyev HJ (2005) A modified Inflammatory Bowel Disease questionnaire and the Vaizey Incontinence questionnaire are simple ways to identify patients with significant gastrointestinal symptoms after pelvic radiotherapy. Br J Cancer 92: 1663-1670.

Peters WA, Liu PY, Barrett 2nd RJ, Stock RJ, Monk BJ, Berek JS, Souhami L, Grigsby P, Gordon W, Alberts DS (2000) Concurrent chemotherapy and pelvic radiation therap compared with pelvic radiation therapy alone as adjuvant therapy after radical surgery in high-risk early-stage cancer of the cervix. J Clin Oncol 18: 1606-1613.

Putta S, Andreyev HJ (2005) Faecal incontinence-a late side effect of pelvic radiotherapy. Clin Oncol ( $R$ Coll Radiol) 17: 469-477.

Rose PG, Bundy BN, Watkins EB, Thigpen JT, Deppe G, Maiman MA, Clarke-Pearson DL, Insalaco S (1999) Concurrent cisplatin-based radiotherapy and chemotherapy for locally advanced cervical cancer. $N$ Eng J Med 340: 1144-1153.

Theis VS, Sripadam R, Ramani V, Lai S (2010) Chronic Radiation Enteritis. Clin Oncol ( $R$ Coll Radiol) 22: 70-83.

Vale CL, Tierney JF, Davidson SE, Drinkwater KJ, Symonds P (2010) Substantial improvement in UK cervical cancer survival with chemoradiotherapy: results of a Royal College of Radiologists' audit. Clin Oncol (R Coll Radiol) 22: 590-601.

Wedlake LJ, Thomas K, Lalji A, Blake P, Khoo VS, Tait D, Andreyev HJ (2010) Predicting late effects of pelvic radiotherapy: is there a better approach? Int J Radiat Oncol Biol Phys 78: 1164-1170.

West CM, Davidson SE (2009) Measurement tools for gastrointestinal symptoms in radiation oncology. Curr Opin Support Palliat Care 3: 36-40.

This work is published under the standard license to publish agreement. After 12 months the work will become freely available and the license terms will switch to a Creative Commons AttributionNonCommercial-Share Alike 3.0 Unported License. 